PROCEEDINGS OF THE

AMERICAN MATHEMATICAL SOCIETY

Volume 129, Number 3, Pages 833-844

S 0002-9939(00)05866-4

Article electronically published on August 30, 2000

\title{
ON FUNCTION AND OPERATOR MODULES
}

\author{
DAVID BLECHER AND CHRISTIAN LE MERDY
}

(Communicated by David R. Larson)

\begin{abstract}
Let $A$ be a unital Banach algebra. We give a characterization of the left Banach $A$-modules $X$ for which there exists a commutative unital $C^{*}$-algebra $C(K)$, a linear isometry $i: X \rightarrow C(K)$, and a contractive unital homomorphism $\theta: A \rightarrow C(K)$ such that $i(a \cdot x)=\theta(a) i(x)$ for any $a \in A, x \in X$. We then deduce a "commutative" version of the Christensen-Effros-Sinclair characterization of operator bimodules. In the last section of the paper, we prove a $w^{*}$-version of the latter characterization, which generalizes some previous work of Effros and Ruan.
\end{abstract}

\section{INTRODUCTION}

Let $H$ be a Hilbert space and let $B(H)$ be the $C^{*}$-algebra of all bounded operators on $H$. Let $A \subset B(H)$ and $B \subset B(H)$ be two unital closed subalgebras and let $X \subset B(H)$ be a closed subspace. If axb belongs to $X$ whenever $a \in A, x \in X$, $b \in B$, then $X$ is called a (concrete) operator $A$-B-bimodule. The starting point of this paper is the abstract characterization of these bimodules due to Christensen, Effros, and Sinclair. Namely, let us consider two unital operator algebras $A$ and $B$ and let $X$ be an arbitrary operator space. Assume that $X$ is an $A$-B-bimodule. Then for any integer $n \geq 1$, the Banach space $M_{n}(X)$ of $n \times n$ matrices with entries in $X$ can be naturally regarded as an $M_{n}(A)-M_{n}(B)$-bimodule, by letting

$$
\left[a_{i k}\right] \cdot\left[x_{k l}\right] \cdot\left[b_{l j}\right]=\left[\sum_{1 \leq k, l \leq n} a_{i k} \cdot x_{k l} \cdot b_{l j}\right]
$$

for any $\left[a_{i k}\right] \in M_{n}(A),\left[x_{k l}\right] \in M_{n}(X),\left[b_{l j}\right] \in M_{n}(B)$. It is shown in CES that if $\|a \cdot x \cdot b\| \leq\|a\|\|x\|\|b\|$ for any $n \geq 1$ and any $a \in M_{n}(A), x \in M_{n}(X)$, and $b \in M_{n}(B)$, then there exist a Hilbert space $H$, and three complete isometries

$$
J: X \rightarrow B(H), \pi_{1}: A \rightarrow B(H), \pi_{2}: B \rightarrow B(H),
$$

such that $\pi_{1}, \pi_{2}$ are homomorphisms and $J(a \cdot x \cdot b)=\pi_{1}(a) J(x) \pi_{2}(b)$ for any $a \in A$, $x \in X, b \in B$. In that case, $X$ is called an (abstract) operator $A$ - $B$-bimodule. Equivalently (in the Operator Space language), operator $A$-B-bimodules $X$ are characterized by the property that the bimodule action $A \times X \times B \longrightarrow X$ extends to a completely contractive map from the Haagerup tensor product $A \otimes_{h} X \otimes_{h} B$ into $X$.

Received by the editors May 24, 1999 .

2000 Mathematics Subject Classification. Primary 47L30, 47L25; Secondary 46H25, 46J10, $46 \mathrm{~L} 07$.

(C)2000 American Mathematical Society 
Let us focus on minimal operator spaces; that is, operator spaces which can be embedded completely isometrically into a commutative $C^{*}$-algebra. First note that if $A$ is a unital operator algebra whose operator space structure is minimal, then it can be represented completely isometrically and algebraically as a unital subalgebra of a commutative unital $C^{*}$-algebra ([B11, Theorem 5]). In that case, $A$ will be called a function algebra. Now if $A, B$ are two function algebras, if $X$ is a minimal operator space, and if $X$ is an operator $A$-B-bimodule, it is natural to wonder whether the corresponding bimodule action can be represented within a commutative $C^{*}$-algebra. We will show in Corollary 2.10 below that this is indeed the case. In other words, the three complete isometries in (1.1) can be chosen to take their values in a common unital commutative $C^{*}$-subalgebra of $B(H)$.

In Section 2 below, in which we prove the latter result, we shall essentially work in a Banach space framework. All Banach spaces considered are complex and are assumed to be different from (0). Given a unital Banach algebra $A$ (with a normalized unit denoted by 1) and a Banach space $X$, we say that $X$ is a left Banach $A$-module if $X$ is equipped with a left module action $A \otimes X \rightarrow X,(a, x) \mapsto a \cdot x$, such that $1 \cdot x=x$ and $\|a \cdot x\| \leq\|a\|\|x\|$ for any $x \in X, a \in A$. Right Banach $B$ modules and Banach $A$ - $B$ bimodules over a pair of unital Banach algebras $(A, B)$ are defined similarly. As seen in [ER1, Section 1], no representation theorem exists for those Banach modules. In Theorem 2.2, we establish a general representation result which partly fills this gap. Namely, let $X$ be a left Banach $A$-module and let $m: A \otimes X \rightarrow X$ be the corresponding module action. We will show that the contractivity of $m$ with respect to various tensor norms on $A \otimes X$ is equivalent to the existence of an isometric embedding $i: X \rightarrow C(K)$ of $X$ into a commutative unital $C^{*}$-algebra $C(K)$ and of a contractive unital homomorphism $\theta: A \rightarrow C(K)$ such that $i(a \cdot x)=\theta(a) i(x)$ for any $a \in A, x \in X$. This result will emphasize the role of the so-called multiplier algebra $\operatorname{Mult}(X)$ of a Banach space $X$, whose definition goes back to $\mathrm{Cu}$ and $\mathrm{AE}$. See also the text $\mathrm{B} 1$ ] (for example pp. 54-55 there). We will derive some simple consequences of known results concerning this algebra.

In Section 3, we continue our investigation of operator bimodules by showing that the Effros-Ruan representation theorem of normal dual operator bimodules over von Neumann algebras (see [ER1]) extends to the non self-adjoint case.

We shall only use a little from Operator Space Theory. Anything undefined here can be found in [B13], [BP], [ER2], or [Pi2]. We also refer the reader to [CS], PS, and CES, for some background on the Haagerup tensor product and operator bimodules, and to [BRS] for the abstract characterization of unital operator algebras.

\section{A Characterization OF FUnCtion modules}

We first fix some tensor norm notation to be used in this section. Given any two Banach spaces $X, Y$, we denote by $Y \otimes X$ their algebraic tensor product. If $\alpha$ is a tensor norm on $Y \otimes X$, the completion of $Y \otimes X$ under $\alpha$ is denoted by $Y \otimes_{\alpha} X$. The Banach space projective (resp. injective) tensor norm is denoted by $\alpha=\wedge$ (resp. $\alpha=\vee$ ). Then following [Pi1, Chapter 2], we denote by $\gamma_{2}$ the norm of factorization through Hilbert space. Given any $1<q \leq \infty$, we let $g_{q}$ be the tensor norm defined in [DF, Section 12.7]. The key fact about this norm is not its definition but its dual relationship with the notion of $p$-summing operators. We recall that given a 
bounded operator $u: X \rightarrow Z$ between Banach spaces, and a number $1 \leq p<\infty$, $u$ is said to be $p$-summing if there exists a constant $K>0$ such that for any finite family $\left(x_{i}\right)_{i}$ in $X, \sum_{i}\left\|u\left(x_{i}\right)\right\|^{p} \leq K^{p} \sup \left\{\sum_{i}\left|\xi\left(x_{i}\right)\right|^{p}: \xi \in X^{*},\|\xi\| \leq 1\right\}$. Moreover the infimum of all numbers $K$ for which this holds is denoted by $\pi_{p}(u)$. We refer e.g. to [Pi1, Chapter 1] for basic information on that notion. Now the fundamental property of $g_{q}$ is the following. Let $1<q \leq \infty$ and $1 \leq p<\infty$ be such that $1 / p+1 / q=1$. Let $T \in\left(Y \otimes_{g_{q}} X\right)^{*}$ and let $u: X \rightarrow Y^{*}$ be the corresponding linear mapping defined by $\langle u(x), y\rangle=T(y \otimes x)$. Then $u$ is $p$-summing and $\pi_{p}(u)=\|T\|$. We finally recall the following contractive embedding, valid for any $1<q \leq 2$ :

$$
Y \otimes_{\wedge} X \subset Y \otimes_{g_{q}} X \subset Y \otimes_{g_{2}} X \subset Y \otimes_{\gamma_{2}} X \subset Y \otimes_{\vee} X
$$

We shall also use the tensor norm $d_{q}$ (see [DF, Section 12.7]). The latter is defined by the isometric identity $Y \otimes_{g_{q}} X=X \otimes_{d_{q}} Y$.

Let $A$ be any unital Banach algebra and let $X$ be a left Banach $A$-module. The module action gives rise to a linear mapping $m: A \otimes X \rightarrow X$ which extends to a contraction from $A \otimes_{\wedge} X$ into $X$. If $\alpha$ is a tensor norm on $A \otimes X$ and if $m$ actually extends to a contraction $A \otimes_{\alpha} X \rightarrow X$, we say that $X$ is a left $\alpha$-module over $A$. When this holds with $\alpha=\vee$, we simply say that $X$ is a left injective module over A. Of course a similar definition can be given for right modules as well. In [T], Tonge characterized function algebras as those unital Banach algebras $A$ for which the multiplication mapping on $A$ extends to a contraction $A \otimes_{g_{q}} A \rightarrow A$, for some $1<q \leq \infty$. Using the main idea of the proof of [T, Theorem 3], we shall give a similar representation result for left $g_{q}$-modules. The relevant definition is the following.

Definition 2.1. Let $X$ be a left Banach $A$-module. We say that $X$ is a (left) function module over $A$, or a (left) function $A$-module, if there is a compact space $K$, a linear isometry $i: X \rightarrow C(K)$, and a contractive unital homomorphism $\theta: A \rightarrow$ $C(K)$, such that $i(a \cdot x)=\theta(a) i(x)$ for any $a \in A, x \in X$. Right function modules are defined similarly.

We warn the reader that our notion of a function module does not coincide with the one defined and studied in [B2] (or the notion in [Cu]).

Before stating our result, we need to introduce a canonical mapping associated to any Banach space. Let $X$ be an arbitrary Banach space. Let $\mathcal{B}$ be the unit ball of $X^{*}$ endowed with the $w^{*}$-topology (so that $\mathcal{B}$ is a compact space), and let $E_{X} \subset \mathcal{B}$ be the subset of its extreme points. Note that $\|\phi\|=1$ for any $\phi \in E_{X}$. We denote by $C_{b}\left(E_{X}\right)$ the unital commutative $C^{*}$-algebra of all bounded continuous functions on $E_{X}$. Then we let

$$
j_{X}: X \longrightarrow C_{b}\left(E_{X}\right)
$$

be the canonical mapping defined by $j_{X}(x)[\phi]=\langle\phi, x\rangle$ for any $x \in X, \phi \in E_{X}$. Then by the Hahn-Banach and Krein-Milman theorems, $j_{X}$ is an isometry.

Theorem 2.2. Let $A$ be a unital Banach algebra and let $X$ be a left Banach $A$ module. The following four assertions are equivalent:

(i) $X$ is a left function module over $A$.

(ii) $X$ is a left injective module over $A$.

(iii) There exists $1<q \leq \infty$ such that $X$ is a left $g_{q}$-module over $A$. 
(iv) There exists a contractive unital homomorphism $\theta: A \rightarrow C_{b}\left(E_{X}\right)$ such that

$$
\forall x \in X, a \in A, \quad j_{X}(a \cdot x)=\theta(a) j_{X}(x) .
$$

Proof. The unital Banach algebra $C_{b}\left(E_{X}\right)$ can be regarded as a $C(K)$-space, with $K$ equal to the Stone-Cech compactification of $E_{X}$. Hence (iv) implies (i). Clearly (ii) implies (iii) (see e.g. (2.1)). Now assume (i); that is, $A$ and $X$ satisfy Definition 2.1. Let $a_{1}, \ldots, a_{n}$ and $x_{1}, \ldots, x_{n}$ be $n$-tuples in $A$ and $X$ respectively. The multiplication mapping on $C(K)$ extends to a contraction on $C(K) \otimes_{\mathrm{V}} C(K)$. Furthermore, $\theta$ and $i$ are both contractions, hence

$$
\left\|\sum_{j=1}^{n} \theta\left(a_{j}\right) i\left(x_{j}\right)\right\|_{C(K)} \leq\left\|\sum_{j=1}^{n} a_{j} \otimes x_{j}\right\|_{A \otimes_{\vee} X}
$$

Since $i$ is an isometry, it follows from the identity $i(a \cdot x)=\theta(a) i(x)$ that the left hand side of (2.3) equals $\left\|m\left(\sum_{j} a_{j} \otimes x_{j}\right)\right\|$. This shows that $\left\|m: A \otimes_{\vee} X \rightarrow X\right\| \leq 1$, whence (ii).

We now proceed to show that (iii) implies (iv), which is the main implication. As mentioned before we use the main idea of Tonge's characterization of function algebras $([\mathrm{T}]$, which in turn was inspired by an argument of Drury.

Let $1<q \leq \infty$ be such that $\left\|m: A \otimes_{g_{q}} X \rightarrow X\right\| \leq 1$ and let $1 \leq p<\infty$ be its conjugate number; that is, $1 / p+1 / q=1$. In the first part of this proof, we give ourselves a fixed $\phi \in E_{X}$ and show that for any $a \in A$ and any $x, y \in X$, we have

$$
\langle\phi, a \cdot x\rangle\langle\phi, y\rangle=\langle\phi, a \cdot y\rangle\langle\phi, x\rangle
$$

As before, we denote by $\mathcal{B}$ the unit ball of $X^{*}$. By our assumption, $m^{*}(\phi)$ is a contractive functional on $A \otimes_{g_{q}} X$; hence the associated linear mapping $u: X \rightarrow A^{*}$ defined by $\langle u(x), a\rangle=\langle\phi, a \cdot x\rangle$ is $p$-summing, with $\pi_{p}(u) \leq 1$. It therefore follows from the Pietsch factorization theorem (see e.g. Pi1, Theorem 1.3]) that there is a Radon probability measure $\mu$ on $\mathcal{B}$ such that

$$
\forall x \in X, \quad\|u(x)\|^{p} \leq \int_{\mathcal{B}}\left|\left\langle x^{*}, x\right\rangle\right|^{p} d \mu\left(x^{*}\right) .
$$

For any $a \in A$, we have $|\langle\phi, a \cdot x\rangle| \leq\|u(x)\|\|a\|$; hence we may deduce from (2.5) that there exists some $F_{a} \in L_{q}(\mathcal{B} ; \mu)$ with norm less than $\|a\|$ such that

$$
\forall x \in X, \quad\langle\phi, a \cdot x\rangle=\int_{\mathcal{B}}\left\langle x^{*}, x\right\rangle F_{a}\left(x^{*}\right) d \mu\left(x^{*}\right) .
$$

Let us simply denote by $F$ the function $F_{a}$ corresponding to the unit $a=1$. Since $1 \cdot x=x$, we obtain

$$
\forall x \in X, \quad\langle\phi, x\rangle=\int_{\mathcal{B}}\left\langle x^{*}, x\right\rangle F\left(x^{*}\right) d \mu\left(x^{*}\right),
$$

with $\|F\|_{L_{q}(\mathcal{B} ; \mu)} \leq 1$. Now using the fact that $\phi$ is an extreme point of $\mathcal{B}$, and following the argument in the proof of [T, Theorem 3], we may deduce from the latter that

$$
\forall x \in X, \quad\langle\phi, x\rangle=\left\langle x^{*}, x\right\rangle F\left(x^{*}\right) \quad \mu-\text { a.e. }
$$


Let $a \in A$ and let $x, y \in X$. Then we can now check (2.4) as follows:

$$
\begin{aligned}
\langle\phi, a \cdot x\rangle\langle\phi, y\rangle & =\int_{\mathcal{B}}\langle\phi, y\rangle\left\langle x^{*}, x\right\rangle F_{a}\left(x^{*}\right) d \mu\left(x^{*}\right) \quad \text { by (2.6), } \\
& =\int_{\mathcal{B}}\left\langle x^{*}, y\right\rangle F\left(x^{*}\right)\left\langle x^{*}, x\right\rangle F_{a}\left(x^{*}\right) d \mu\left(x^{*}\right) \quad \text { by }(2.7), \\
& =\int_{\mathcal{B}}\langle\phi, x\rangle\left\langle x^{*}, y\right\rangle F_{a}\left(x^{*}\right) d \mu\left(x^{*}\right) \quad \text { by }(2.7), \\
& =\langle\phi, a \cdot y\rangle\langle\phi, x\rangle \quad \text { by }(2.6) .
\end{aligned}
$$

For any $a \in A$, we may now define a function $\theta(a): E_{X} \rightarrow \mathrm{C}$ as follows. Given any $\phi \in E_{X}$, we pick $x \in X$ so that $\langle\phi, x\rangle \neq 0$ (recall that $\phi \neq 0$ ) and we set

$$
\theta(a)[\phi]=\frac{\langle\phi, a \cdot x\rangle}{\langle\phi, x\rangle} .
$$

Indeed it follows from (2.4) that this definition does not depend on the choice of $x$. Clearly $\theta(a)$ is a continuous function. Moreover since any $\phi \in E_{X}$ has norm 1, we have

$$
\begin{aligned}
|\theta(a)[\phi]| & \leq \inf \{|\langle\phi, a \cdot x\rangle|: x \in X,|\langle\phi, x\rangle|=1\} \\
& \leq\|a\| \inf \{\|x\|: x \in X,|\langle\phi, x\rangle|=1\}=\|a\| .
\end{aligned}
$$

This shows that $\theta(a) \in C_{b}\left(E_{X}\right)$ for any $a \in A$ and that $\theta: A \rightarrow C_{b}\left(E_{X}\right)$ is a contractive linear mapping. Moreover (2.4) can now be re-written as follows:

$$
\forall a \in A, x \in X, \phi \in E_{X}, \quad \theta(a)[\phi]\langle\phi, x\rangle=\langle\phi, a \cdot x\rangle .
$$

If $a, b \in A$, we see by applying (2.8) three times that for any $\phi \in E_{X}$ and $x \in X$, we have

$$
\begin{aligned}
\theta(a b)[\phi]\langle\phi, x\rangle & =\langle\phi, a b \cdot x\rangle=\langle\phi, a \cdot(b \cdot x)\rangle \\
& =\theta(a)[\phi]\langle\phi, b \cdot x\rangle \\
& =\theta(a)[\phi] \theta(b)[\phi]\langle\phi, x\rangle .
\end{aligned}
$$

This shows that $\theta$ is a homomorphism. The latter is clearly unital. Since (2.2) follows from (2.8), the proof is complete.

Remark 2.3. We may obviously write a version of Theorem 2.2 for right modules. Namely, let $B$ be a unital Banach algebra and let $X$ be a right Banach $B$-module. Then $X$ is a right function module over $B$ if and only if $X$ is a right injective module over $B$ if and only if there exists $1<q \leq \infty$ such that $X$ is a right $d_{q}$-module over $B$ if and only if there exists a contractive unital homomorphism $\theta: B \rightarrow C_{b}\left(E_{X}\right)$ such that $j_{X}(x \cdot b)=j_{X}(x) \theta(b)$ for any $x \in X, b \in B$.

As an application of the fact that we may use the same fixed embedding $j_{X}$ in Theorem 2.2 and Remark 2.3, we get the following observation concerning bimodules.

Remark 2.4. Let $A, B$ be two unital Banach algebras and assume that $X$ is both a left function $A$-module and a right function $B$-module. Then there exist two contractive unital homomorphisms $\theta_{1}: A \rightarrow C_{b}\left(E_{X}\right)$ and $\theta_{2}: B \rightarrow C_{b}\left(E_{X}\right)$ such that $j_{X}(a \cdot x)=\theta_{1}(a) j_{X}(x)$ and $j_{X}(x \cdot b)=j_{X}(x) \theta_{2}(b)$ for any $a \in A, x \in X, b \in B$. We may then write

$$
j_{X}((a \cdot x) \cdot b)=\theta_{1}(a) j_{X}(x) \theta_{2}(b)=j_{X}(a \cdot(x \cdot b)) .
$$


Since $j_{X}$ is $1-1$, we deduce the associativity condition

$$
\forall a \in A, x \in X, b \in B, \quad(a \cdot x) \cdot b=a \cdot(x \cdot b) .
$$

In other words the left $A$-module and right $B$-module actions on $X$ automatically induce an $A$ - $B$-bimodule action on $X$.

Remark 2.5. (1) It is fairly easy to recover Tonge's characterization of function algebras from our Theorem 2.2. Indeed, let $1<q \leq \infty$ be a number and let $A$ be a unital Banach algebra. Assume that the multiplication mapping $A \otimes A \rightarrow A$ extends to a contraction from $A \otimes_{g_{q}} A$ into $A$, and apply Theorem 2.2 with $X=A$. Let $\theta: A \rightarrow C_{b}\left(E_{A}\right)$ be the contractive unital homomorphism given by the latter. For any $a \in A$, we have $j_{A}(a)=\theta(a) j_{A}(1)$; hence

$$
\|a\|=\left\|j_{A}(a)\right\|=\left\|\theta(a) j_{A}(1)\right\| \leq\|\theta(a)\|\left\|j_{A}(1)\right\| \leq\|\theta(a)\| .
$$

This shows that $\theta$ is actually an isometric unital homomorphism; hence $A$ is a function algebra.

(2) A "nonassociative" version of Tonge's result can be derived from the observation made in Remark 2.4. Namely, let $A$ be a Banach space and assume that there is a bilinear map $m: A \times A \rightarrow A$ and an element $e \in A$ such that $\|e\|=1$ and $m(a, e)=m(e, a)=a$ for any $a \in A$. Assume that for some $1<q_{1}, q_{2} \leq \infty$, we both have

$$
\left\|m: A \otimes_{g_{q_{1}}} A \rightarrow A\right\| \leq 1 \quad \text { and } \quad\left\|m: A \otimes_{d_{q_{2}}} A \rightarrow A\right\| \leq 1
$$

Then $m$ is actually a multiplication on $A$, and $A$ equipped with $m$ is a function algebra.

Indeed, the proof of (2.8) above does not use the algebraic structure of $A$; hence under the assumption (2.9), it shows the existence of contractive linear maps $\theta_{1}, \theta_{2}: A \rightarrow C_{b}\left(E_{A}\right)$ such that $\theta_{1}(a) j_{A}(b)=j_{A}(m(a, b))=j_{A}(a) \theta_{2}(b)$ for any $a, b \in A$. Arguing as in Remark 2.4, we deduce the associativity condition $m(m(a, c), b)=m(a, m(c, b))$. This should be compared with [BRS, Corollary 2.4].

For any Banach space $X$, let us consider the multiplier algebra of $X([\mathrm{AE}], \mathrm{Cu}])$ defined by

$$
\operatorname{Mult}(X)=\left\{f \in C_{b}\left(E_{X}\right): f j_{X}(X) \subset j_{X}(X)\right\} .
$$

Clearly $\operatorname{Mult}(X) \subset C_{b}\left(E_{X}\right)$ is a function algebra. We refer e.g. to [B1, pp. 54$55]$ for basic properties and an alternate definition of this algebra. It follows from Theorem 2.2 that for any unital Banach algebra $A$, there is a 1-1 correspondence between function $A$-modules actions on $X$ and contractive unital homomorphisms from $A$ into $\operatorname{Mult}(X)$. Let us give a list of instructive examples and observations, partly based on former results concerning multiplier algebras ([B1], B2], [J]).

Examples 2.6. (1) We let $A$ be a unital Banach algebra. If $\varphi \in A^{*}$ is a character of $A$, we may define an $A$-module action on any $X$ by letting $a \cdot x=\varphi(a) x$. Then $X$ is an function $A$-module. Such a function module will be called elementary. Conversely, if $\theta: A \rightarrow \operatorname{Mult}(X)$ is a contractive unital homomorphism and if $\Psi$ is any character of $\operatorname{Mult}(X)$, then $\theta^{*}(\Psi)$ is a character of $A$. We deduce that the following are equivalent:

(i) $A$ admits characters.

(ii) There exists a nonzero function $A$-module.

(iii) Any Banach space $X$ admits a function $A$-module action. 
(2) Let $X$ be a Banach space. If $\operatorname{Mult}(X)=\mathrm{C}$, then any function module action on $X$ is elementary. This holds for instance if $X$ is strictly convex ([J] Corollary $3]$ ), or if $X$ is smooth ([B2, Proposition 5.2]), or if $X$ is an $L_{1}$-space ([B2, p. 136]).

(3) Let $X$ be a Banach space. If $X$ does not contain any isometric copy of $c_{0}$, then $\operatorname{Mult}(X)$ is finite dimensional $([\bar{J}])$. This holds for instance if $X$ is reflexive. Assuming this, let $n$ be the dimension of $\operatorname{Mult}(X)$. Then $\operatorname{Mult}(X)=\ell_{\infty}^{n}$; hence there exist subspaces $X_{1}, \ldots, X_{n}$ of $X$ such that $\operatorname{dim}\left(\operatorname{Mult}\left(X_{j}\right)\right)=1$ for each $1 \leq$ $j \leq n$ and $X$ is equal to the $\ell_{\infty} \operatorname{direct} \operatorname{sum} X=X_{1} \stackrel{\infty}{\oplus} \cdots \stackrel{\infty}{\oplus} X_{n}$ ([B2, Proposition 5.1]). This implies that if $A$ is a unital Banach algebra admitting characters, the datum of a function $A$-module action on $X$ is equivalent to the datum of elementary actions of $A$ on each $X_{j}$. More precisely, let $\varphi_{1}, \ldots, \varphi_{n}$ be characters on $A$. We may define a function $A$-module action on $X$ by letting $a \cdot\left(x_{1}+\cdots+x_{n}\right)=\varphi_{1}(a) x_{1}+\cdots+\varphi_{n}(a) x_{n}$ for any $a \in A, x_{1} \in X_{1}, \ldots, x_{n} \in X_{n}$. And conversely, any function $A$-module action on $X$ arises in that manner.

(4) Let $X=B$ be a function algebra. Then $\operatorname{Mult}(B)=B$ ([B2, p. 123]). Thus for any unital Banach algebra $A$, there is a 1-1 correspondence between $A$ module action on $B$ and contractive unital homomorphisms $\theta: A \rightarrow B$. Given such a mapping $\theta$, the corresponding action is given by $a \cdot b=\theta(a) b$ for $a \in A, b \in B$.

(5) Let $A$ be the disc algebra and let $X$ be any Banach space. Then the function $A$-module actions on $X$ are in an obvious correspondence with elements of the closed unit ball of $\operatorname{Mult}(X)$.

(6) The most interesting examples of function modules are perhaps those obtained by the following process. Let $K$ be a compact space and let $A \subset C(K)$ be a function algebra. Then for any set $S \subset C(K)$, the Banach space $X=\overline{A S}=$ $\overline{\operatorname{Span}}\{a s: a \in A, s \in S\}$ is a left function module over $A$.

Remark 2.7. If $A, B$ are unital Banach algebras, if $X$ is a left Banach $A$-module, and if $\rho: B \rightarrow A$ is a contractive unital homomorphism, then $X$ becomes a left Banach $B$-module in a canonical way; namely $b \cdot x=\rho(b) \cdot x$. We shall call this a prolongation of the $A$-module action. Obviously, any prolongation of a function module is a function module. In fact, for any $X$, the multiplication in $C_{b}\left(E_{X}\right)$ makes $X$ a left function module over $\operatorname{Mult}(X)$, and every function module action on $X$ is a prolongation of this one.

We now turn to operator modules and operator bimodules in the sense of [CES]. Recall that the tensor norms $\gamma_{2}, d_{2}$, and $g_{2}$ are related to the Haagerup tensor product of operator spaces as follows. If $X$ and $Y$ are two Banach spaces, then the following isometric equalities hold:

$$
\begin{gathered}
M I N(Y) \otimes_{h} M I N(X)=Y \otimes_{\gamma_{2}} X ; \\
M A X(Y) \otimes_{h} M I N(X)=Y \otimes_{g_{2}} X ; \quad M I N(Y) \otimes_{h} M A X(X)=Y \otimes_{d_{2}} X .
\end{gathered}
$$

See e.g. [Bl2 Theorem 3.1]. Applying Theorem 2.2 and Remarks 2.3 and 2.4, we therefore obtain the following.

Corollary 2.8. Let $A$ and $B$ be two unital operator algebras, and let $X$ be a Banach $A$-B-bimodule. Let $m: A \otimes X \otimes B \rightarrow X, m_{1}: A \otimes X \rightarrow X$ and $m_{2}: X \otimes B \rightarrow X$ be linearly defined by the module actions. The following assertions are equivalent:

(i) There exist two numbers $1<q_{1}, q_{2} \leq \infty$ such that

$$
\left\|m_{1}: A \otimes_{g_{q_{1}}} X \longrightarrow X\right\| \leq 1 \quad \text { and } \quad\left\|m_{2}: X \otimes_{d_{q_{2}}} B \longrightarrow X\right\| \leq 1 .
$$


(ii) We have $\left\|m: M A X(A) \otimes_{h} M I N(X) \otimes_{h} M A X(B) \longrightarrow M I N(X)\right\|_{c b} \leq 1$.

(iii) The operator space $M I N(X)$ is an operator $A$-B-bimodule.

(iv) We have $\left\|m_{1}: A \otimes_{\vee} X \longrightarrow X\right\| \leq 1$ and $\left\|m_{2}: X \otimes_{\vee} B \longrightarrow X\right\| \leq 1$.

(v) There exist two contractive unital homomorphisms $\theta_{1}: A \rightarrow C_{b}\left(E_{X}\right), \theta_{2}: B \rightarrow$ $C_{b}\left(E_{X}\right)$ such that

$$
\forall(a, x, b) \in A \times X \times B, \quad j_{X}(a \cdot x \cdot b)=\theta_{1}(a) j_{X}(x) \theta_{2}(b) .
$$

Using part (1) in Example 2.6, we deduce an obstruction for a minimal operator space to be an operator module.

Corollary 2.9. Let $A$ be an operator algebra without characters (for instance, let $A=B(H)$, with $\operatorname{dim}(H) \geq 2$ ). Then no minimal operator space (except (0)) can be an operator A-module.

We now restrict to the case of function algebras, on which characters exist. We obtain the following "commutative" version of the Christensen-Effros-Sinclair characterization of operator bimodules.

Corollary 2.10. Let $A$ and $B$ be two function algebras, and let $X$ be a Banach $A$-B-bimodule. The following assertions are equivalent:

(i) The operator space $M I N(X)$ is an operator $A$-B-bimodule.

(ii) There exist a unital commutative $C^{*}$-algebra $\mathcal{C}$, two isometric unital homomorphisms $\pi_{1}: A \rightarrow \mathcal{C}, \pi_{2}: B \rightarrow \mathcal{C}$, and a linear isometry $J: X \rightarrow \mathcal{C}$ such that $J(a \cdot x \cdot b)=\pi_{1}(a) J(x) \pi_{2}(b)$ for any $a \in A, x \in X, b \in B$.

Proof. Assume (i). By Corollary 2.8, there exist two contractive unital homomorphisms $\theta_{1}: A \rightarrow C_{b}\left(E_{X}\right), \theta_{2}: B \rightarrow C_{b}\left(E_{X}\right)$ such that (2.10) holds. Since $A$ and $B$ are function algebras, we may find unital commutative $C^{*}$-algebras $C_{1}, C_{2}$, and isometric unital homomorphisms $\rho_{1}: A \rightarrow C_{1}, \rho_{2}: B \rightarrow C_{2}$. We also give ourselves two characters $\varphi_{1} \in A^{*}, \varphi_{2} \in B^{*}$.

We let $\mathcal{C}=C_{1} \stackrel{\infty}{\oplus} C_{b}\left(E_{X}\right) \stackrel{\infty}{\oplus} C_{2}$ be the $\ell_{\infty}$-direct sum of our three unital commutative $C^{*}$-algebras. Then $\mathcal{C}$ is a unital commutative $C^{*}$-algebra as well. We now define $J: X \rightarrow \mathcal{C}, \pi_{1}: A \rightarrow \mathcal{C}$ and $\pi_{2}: B \rightarrow \mathcal{C}$ by letting $J(x)=0 \oplus j_{X}(x) \oplus 0$, $\pi_{1}(a)=\rho_{1}(a) \oplus \theta_{1}(a) \oplus \varphi_{1}(a) 1$, and $\pi_{2}(b)=\varphi_{2}(b) 1 \oplus \theta_{2}(b) \oplus \rho_{2}(b)$. It is easy to check, using (2.10), that these mappings satisfy (ii).

Remark 2.11. Clearly submodules of function modules, $L_{\infty}$-direct sums of function modules, and prolongations of function modules (see Remark 2.7) are function modules.

Let $A$ be an operator algebra. The category ${ }_{A} F M O D$ of function $A$-modules is a full subcategory of ${ }_{A} O M O D$, the operator $A$-modules. The relationship of the class of function $A$-modules, to the class ${ }_{A} O M O D$, seems to resemble the relationship of function algebras to operator algebras. That is, unlike ${ }_{A} O M O D,{ }_{A} F M O D$ is not closed with respect to certain elementary constructions. For example the quotient of a function module by a closed submodule (which could perhaps be called a $Q$ module) is an operator $A$-module, but not necessarily a function $A$-module. The module Haagerup tensor product of two function modules (which one may view as an appropriate quotient of the $\gamma_{2}$ tensor product) is not a function $A$-module (but is an operator $A$-module). This is also connected with the fact that the category of minimal operator spaces is not closed under some basic operator space constructions. 
Some of the ideas here also led to the work $\mathrm{B} 15$. In that paper singly generated function modules are studied in a little more detail.

\section{A Result on normal DUAl operator Bimodules}

The Christensen-Effros-Sinclair representation theorem for operator $A$ - $B$-bimodules (reviewed in Section 1 above) is stated and proved in [CES] for unital $C^{*}$-algebras $A$ and $B$. However it is well known that their result (namely CES, Corollary 3.3]) extends with the same proof to the case when $A$ and $B$ are merely non-self-adjoint unital operator algebras. In ER1], a version of [CES, Corollary $3.3]$ is proved for the so-called normal dual operator bimodules over a pair of von Neumann algebras $(A, B)$. The aim of this section is to extend that result to the case when $A$ and $B$ are only assumed to be unital dual operator algebras. The new ingredient used to treat this case is the $w^{*}$-version of Wittstock's representation theorem for $w^{*}$-continuous completely bounded maps on dual operator algebras, recently established in [L2].

Let us briefly recall or introduce a few definitions. An operator space $X$ is called a dual operator space if there is another operator space $Y$ such that $X=Y^{*}$ completely isometrically. In that case there exists a $w^{*}$-continuous completely isometric embedding of $X$ into some $B(H)$ (see [B13] or [ER1]). Accordingly, an operator algebra $A$ is called a dual operator algebra if it can be represented algebraically and completely isometrically as a $w^{*}$-closed subalgebra of $B(H)$ for some Hilbert space $H$. We refer to [L1] for an abstract characterization of dual operator algebras.

Let $A$ and $B$ be two unital dual operator algebras and let $X$ be a dual operator space. Assume that $X$ is an operator $A$-B-bimodule. Following [ER1], we say that $X$ is a normal dual operator $A$ - $B$-bimodule if in addition, the trilinear mapping

$$
A \times X \times B \longrightarrow X, \quad(a, x, b) \mapsto a \cdot x \cdot b
$$

is separately $w^{*}$-continuous. The following is a representation theorem for those bimodules. It extends [ER1, Theorem 3.4] and [ER1, Theorem 4.1].

Theorem 3.1. Let $A$ and $B$ be two unital dual operator algebras and let $X$ be a normal dual operator $A$-B-bimodule.

(1) There exist a Hilbert space $H, a w^{*}$-continuous complete isometry $J: X \rightarrow$ $B(H)$, and $w^{*}$-continuous completely isometric homomorphisms $\pi_{1}: A \rightarrow$ $B(H), \pi_{2}: B \rightarrow B(H)$ such that $J(a \cdot x \cdot b)=\pi_{1}(a) J(x) \pi_{2}(b)$ for any $a \in A$, $x \in X, b \in B$.

(2) If $A=B$, we may find a $w^{*}$-continuous and completely isometric unital homomorphism $\pi: A \rightarrow B(H)$ such that (1) holds with $\pi_{1}=\pi_{2}=\pi$.

Proof. Let $m: A \otimes_{h} X \otimes_{h} B \rightarrow X$ be the completely contractive mapping induced by the bimodule action. We let $j: X \rightarrow B(K)$ be a $w^{*}$-continuous completely isometric embedding for some Hilbert space $K$. Then $j m$ is completely contractive; hence by the factorization theorem of multilinear completely bounded maps ([CS], [PS $)$, there exist two Hilbert spaces $E, F$, and three completely contractive maps $\alpha: A \rightarrow B(E, K), v: X \rightarrow B(F, E)$, and $\beta: B \rightarrow B(K, F)$, such that

$$
\forall a \in A, x \in X, b \in B, \quad j(a \cdot x \cdot b)=\alpha(a) v(x) \beta(b) .
$$

A key fact is that since $m$ is assumed to be separately $w^{*}$-continuous and $j$ is $w^{*}$ continuous, it follows from [ER3, Theorem 3.1] that our three maps $\alpha, v, \beta$ can be chosen to be $w^{*}$-continuous. By [L2, Proposition 3.4], we can then factor $\alpha$ and $\beta$ 
as follows. There exist a Hilbert space $H_{1}$, a $w^{*}$-continuous unital homomorphism $\theta_{1}: A \rightarrow B\left(H_{1}\right)$ and two contractions $E \stackrel{S}{\longrightarrow} H_{1} \stackrel{R}{\longrightarrow} K$ such that $\alpha(a)=R \theta_{1}(a) S$. Likewise, there exist a Hilbert space $H_{2}$, a $w^{*}$-continuous unital homomorphism $\theta_{2}: B \rightarrow B\left(H_{2}\right)$ and two contractions $K \stackrel{U}{\longrightarrow} H_{2} \stackrel{T}{\longrightarrow} F$ such that $\beta(b)=T \theta_{2}(b) U$. Moreover, changing $H_{1}$ and $H_{2}$ to subspaces if necessary, we may assume (as in the proof of [L2, Proposition 3.4]) that

$$
H_{1}=\overline{\operatorname{Span}}\left\{\theta_{1}(A)^{*} R^{*}(K)\right\} \quad \text { and } \quad H_{2}=\overline{\operatorname{Span}}\left\{\theta_{2}(B) U(K)\right\} .
$$

Let us now define $u: X \rightarrow B\left(H_{2}, H_{1}\right)$ by letting $u(x)=S v(x) T$. By construction, $u$ is $w^{*}$-continuous and completely contractive. Moreover we see from (3.1) that

$$
\forall a \in A, x \in X, b \in B, \quad j(a \cdot x \cdot b)=R \theta_{1}(a) u(x) \theta_{2}(b) U .
$$

Applying (3.3) twice it is fairly easy to see that for any $a^{\prime}, a \in A, x \in X, b, b^{\prime} \in B$, we have

$$
R \theta_{1}\left(a^{\prime}\right) \theta_{1}(a) u(x) \theta_{2}(b) \theta_{2}\left(b^{\prime}\right) U=R \theta_{1}\left(a^{\prime}\right) u(a \cdot x \cdot b) \theta_{2}\left(b^{\prime}\right) U .
$$

It therefore follows from (3.2) that for any $a \in A, x \in X, b \in B$, we have

$$
u(a \cdot x \cdot b)=\theta_{1}(a) u(x) \theta_{2}(b) .
$$

Let $H=H_{1} \stackrel{2}{\oplus} H_{2}$ be the Hilbert direct sum of $H_{1}$ and $H_{2}$, and let $\pi_{1}: A \rightarrow B(H)$, $\pi_{2}: B \rightarrow B(H)$, and $J: X \rightarrow B(H)$ be defined by letting

$$
J(x)=\left(\begin{array}{cc}
0 & u(x) \\
0 & 0
\end{array}\right), \quad \pi_{1}(a)=\left(\begin{array}{cc}
\theta_{1}(a) & 0 \\
0 & 0
\end{array}\right), \quad \pi_{2}(b)=\left(\begin{array}{cc}
0 & 0 \\
0 & \theta_{2}(b)
\end{array}\right) .
$$

Clearly $\pi_{1}$ and $\pi_{2}$ are $w^{*}$-continuous completely contractive homomorphisms. Moreover the identity (3.4) implies that

$$
\forall a \in A, x \in X, b \in B, \quad J(a \cdot x \cdot b)=\pi_{1}(a) J(x) \pi_{2}(b) .
$$

Applying (3.3) with $a=1$ and $b=1$, we see that $u$ is actually a complete isometry; hence $J$ is a $w^{*}$-continuous complete isometry. The assertion (1) is thus proved, up to the fact that $\pi_{1}$ and $\pi_{2}$ are merely completely contractive instead of being completely isometric. To get this completely isometric condition, it suffices to apply a direct sum argument as e.g. in the proof of Corollary 2.10 above.

We now turn to the proof of (2). Assume that $A=B$ and that $\theta_{1}, \theta_{2}$ and $u$ are constructed as above. Again we let $H=H_{1} \stackrel{2}{\oplus} H_{2}$, and this time we define $\pi: A \rightarrow B(H)$ and $J: X \rightarrow B(H)$ by letting

$$
J(x)=\left(\begin{array}{cc}
0 & u(x) \\
0 & 0
\end{array}\right), \quad \pi(a)=\left(\begin{array}{cc}
\theta_{1}(a) & 0 \\
0 & \theta_{2}(a)
\end{array}\right) .
$$

Then $\pi$ is a $w^{*}$-continuous completely contractive unital homomorphism and (3.5) holds with $\pi_{1}=\pi_{2}=\pi$. We can thus conclude as in the proof of (1).

Remark 3.2. In [B14 Theorem 2.2], the first author gave a completely isomorphic version of the Christensen-Effros-Sinclair representation theorem, as follows. Let $(A, B)$ be a pair of (possibly nonunital) operator algebras, and let $X$ be an operator space. Assume that $A$ is an $A$ - $B$-bimodule and that the bimodule action is completely bounded from $A \otimes_{h} X \otimes_{h} B$ into $X$. Then one can find complete isomorphisms $J: X \rightarrow B(H), \pi_{1}: A \rightarrow B(H)$ and $\pi_{2}: B \rightarrow B(H)$ such that $\pi_{1}, \pi_{2}$ are homomorphisms and $J(a \cdot x \cdot b)=\pi_{1}(a) J(x) \pi_{2}(b)$ for any $a \in A, x \in X, b \in B$. Arguing as in the proof of [B14, Theorem 2.2] and using the completely isomorphic 
characterization of dual operator algebras given in [L1 Theorem 7], one can show that if $X$ is a dual operator space, if $A$ and $B$ are dual operator algebras, and if the bimodule action is separately $w^{*}$-continuous, then that representation can be achieved with the extra condition that $\pi_{1}, \pi_{2}$, and $J$ are $w^{*}$-continuous. We leave the details to the reader.

Remark 3.3. Comparing our results in Sections 2 and 3, it is tempting to try to study an appropriate notion of dual function module. The first question arising here is the following apparently open problem. Let $A$ be a function algebra and assume that $A$ is a dual space. Does there exist a $w^{*}$-continuous isometric unital homomorphism of $A$ into some $L_{\infty}$-space?

\section{ACKNOWLEDGMENTS}

This paper was written while the second author was visiting the University of Houston. He wishes to thank the Department of Mathematics of UH for its warm hospitality.

\section{REFERENCES}

[AE] E.M. Alfsen and E.G. Effros, Structure in real Banach spaces I, II, Annals of Math. 96 (1972), 98-173. MR 50:5432

[B1] E. Behrends, M-structure and the Banach-Stone theorem, Springer Lecture Notes 736, Berlin-Heidelberg-New-York, 1979. MR 81b:46002

[B2] E. Behrends, Multiplier representations and an application to the problem whether $A \otimes_{\varepsilon} X$ determines $A$ and/or $X$, Math. Scand. 52 (1983), 117-144. MR 84m:46050

[Bl1] D.P. Blecher, Commutativity in operator algebras, Proc. Amer. Math. Soc 109 (1990), 709-715. MR 90k:46128

[B12] D.P. Blecher, Tensor products of operator spaces II, Canad. J. Math. 44 (1992), 75-90. MR 93e: 46084

[B13] D.P. Blecher, The standard dual of an operator space, Pacific J. Math. 153 (1992), 15-30. MR 93d: 47083

[Bl4] D.P. Blecher, A generalization of Hilbert modules, J. Funct. Anal. 136 (1996), 365-421. MR 97g:46071

[B15] D.P. Blecher, The Shilov boundary of an operator space - and the characterization theorems, Preprint (1999).

[BP] D.P. Blecher and V.I. Paulsen, Tensor products of operator spaces, J. Funct. Anal. 99 (1991), 262-292. MR 93d:46095

[BRS] D.P. Blecher, Z.-J. Ruan and A.M. Sinclair, A characterization of operator algebras, J. Funct. Anal. 89 (1990), 188-201. MR 91b:47098

[CES] E. Christensen, E.G. Effros and A.M. Sinclair, Completely bounded multilinear maps and $C^{*}$-algebraic cohomology, Invent. Math. 90 (1987), 279-296. MR 89k:46084

[CS] E. Christensen and A.M. Sinclair, Representation of completely bounded multilinear operators, J. Funct. Anal. 72 (1987), 151-181. MR 89f:46113

$[\mathrm{Cu}]$ F. Cunningham, M-structure in Banach spaces, Math. Proc. Camb. Philos. Soc. 63 (1967), 613-629. MR 35:3415

[DF] A. Defant and K. Floret, Tensor norms and operator ideals, North-Holland, Amsterdam, 1993. MR 94e:46130

[ER1] E.G. Effros and Z.-J. Ruan, Representations of operator bimodules and their applications, J. Operator Theory 19 (1988), 137-157. MR 91e:46077]

[ER2] E.G. Effros and Z.-J. Ruan, A new approach to operator spaces, Canadian Math. Bull. 34 (1991), 329-337. MR 93a:47045

[ER3] E.G. Effros and Z.-J. Ruan, Operator convolution algebras: an approach to quantum groups, Unpublished (1991).

[J] K. Jarosz, Multipliers in complex Banach spaces and structure of the unit balls, Studia Math. T. LXXXVII (1987), 197-213. MR 89j:46017 
[L1] C. Le Merdy, An operator space characterization of dual operator algebras, Amer. J. Math. 121 (1999), 55-63. CMP 99:16

[L2] C. Le Merdy, Finite rank approximation and semidiscreteness for linear operators, Annales Inst. Fourier 49 (1999), 1869-1901. CMP 2000:08

[PS] V.I. Paulsen and R.R. Smith, Multilinear maps and tensor norms on operator systems, J. Funct. Anal. 73 (1987), 258-276. MR 89m:46099

[Pi1] G. Pisier, Factorization of linear operators and geometry of Banach spaces, CBMS Series 60 (Amer. Math. Soc., Providence, R.I.), 1986. MR 88a:47013

[Pi2] G. Pisier, An introduction to the theory of operator spaces, Preprint (1997).

[T] A.M. Tonge, Banach algebras and absolutely summing operators, Math. Proc. Cambridge Philos. Soc. 80 (1976), 465-473. MR 55:11071

Department of Mathematics, University of Houston, Houston, Texas 77204-3476

E-mail address: dblecher@math.uh.edu

Département de Mathématiques, Université de Franche-Comté, 25030 Besançon Cedex, France 